\title{
Formation of hot-electron ensembles quasiequilibrated in momentum space by ultrafast momentum scattering of highly excited hot electrons photoinjected into the $\Gamma$ valley of GaAs
}

\author{
Hiroshi Tanimura, ${ }^{*}$ Jun'ichi Kanasaki, and Katsumi Tanimura \\ The Institute of Scientific and Industrial Research, Osaka University, 8-1 Mihogaoka, Ibaraki, Osaka 567-0047, Japan \\ Jelena Sjakste and Nathalie Vast \\ Ecole Polytechnique, Laboratoire des Solides Irradiés, Route de Saclay, CNRS UMR 7642, CEA-DFR-IRAMIS, \\ Université Paris-Saclay, F-91120 Palaiseau, France \\ Matteo Calandra and Francesco Mauri ${ }^{\dagger}$ \\ IMPMC, UMR CNRS 7590, Sorbonne Universités-UPMC Université Paris 6, MNHN, IRD, 4 place Jussieu, F-75005 Paris, France
}

(Received 11 September 2015; revised manuscript received 6 March 2016; published 6 April 2016)

\begin{abstract}
We study ultrafast scattering dynamics of hot electrons photoinjected with high excess energies in the $\Gamma$ valley of the conduction band of GaAs, using time- and angle-resolved photoemission spectroscopy and $a b$ initio calculations. At ultrafast rates of the order of $10 \mathrm{fs}$, the packets in the $\Gamma$ valley are transformed into hot-electron ensembles (HEEs) quasiequilibrated in momentum space but not in energy space. The energy relaxation of the HEEs takes place as a whole on a longer time scale with rates dependent only on the excess energy, irrespective of the momenta of hot electrons. Both momentum scattering and energy relaxation are ruled by the electron-phonon interaction.
\end{abstract}

DOI: 10.1103/PhysRevB.93.161203

Ultrafast scattering of energetic carriers in semiconductors has been a strategic research field for the past two decades, since it not only constitutes the key process that determines functional limits and properties of micro-, nano-, and optoelectronics, but because it also provides one of the best systems to study ultrafast relaxation of nonequilibrated states in solids [1-3]. An interband excitation with ultrashort light pulses having broadband coherence yields wave packets consisting of Bloch's functions in the conduction (CB) and valence bands [1-4]. The ultrafast scattering processes in the coherence regime [1], leading to the decay of the wave packets, is crucial not only to elucidate dynamical coherence effects such as Bloch oscillations [1-3,5], but to identify fundamental pathways of hot-electron relaxation in the $\mathrm{CB}$ with multivalley structures. For the excited states confined within the central $\Gamma$ valley, the electron-electron $(e-e)$ interaction is dominant in the initial momentum scattering processes because of the low rates of electron-phonon ( $e-\mathrm{ph})$ interactions [1-3]. However, the scattering dynamics depends critically on the excess energy $\varepsilon$ of hot electrons as the modes and rates of scattering processes induced by $e$-ph interactions change drastically depending on $\varepsilon$. Despite decades of extensive studies [1-3], clear understanding of ultrafast scattering processes of hot electrons with high excess energies remains elusive due to the high complexity of the problem and partly to methodological limitations in capturing the scattering dynamics unambiguously.

Here we study the ultrafast dynamics of hot electrons photoinjected into the $\Gamma$ valley with high excess energies in $\mathrm{GaAs}$, a prototypical direct-gap semiconductor, by combining

\footnotetext{
*Present address: Research Center for Ultra-High Voltage Electron Microscopy, Osaka University, 7-1 Mihogaoka, Osaka 567-0047, Japan.

†Present address: Dipartimento di Fisica, Università di Roma La Sapienza, Piazzale Aldo Moro 5, I-00185 Roma, Italy.
}

time- and angle-resolved photoemission spectroscopy with state of-the-art $a b$ initio theoretical calculations. The former directly captures ultrafast changes of electron distribution functions resolved in energy and momentum space [6,7], which provide the key information necessary to elucidating incoherent scattering dynamics [2], while the latter enables us to perform parameter-free computation of $e$-ph scattering rates [8-10]. We reveal that the packets photoinjected into the $\Gamma$ valley are transformed, within a few tens of fs, into the hot-electron ensembles (HEEs), which are quasiequilibrated in momentum space but still highly nonthermal in energy space. Energy relaxation of the HEEs takes place as a whole at longer time domains, with a rate ruled by the excess energy only. The $e$-ph interaction entirely governs these ultrafast processes for excitation density $\rho$ less than $5 \times 10^{17} \mathrm{~cm}^{-3}$.

Zn-doped $p$-type GaAs wafers (carrier concentration $1.5 \times 10^{17} \mathrm{~cm}^{-3}$ ) were cleaved under ultrahigh vacuum conditions $\left(<5 \times 10^{-11}\right.$ Torr). Atomic structures of the (110) surfaces were characterized in situ by a scanning tunneling microscope. The Ti-sapphire laser system used and the experimental geometry were described in Ref. [6]. Briefly, pump pulses were 40-fs pulses centered at photon energies between 1.6 and $2.4 \mathrm{eV}$ (the band-gap energy is $1.43 \mathrm{eV}$ at $300 \mathrm{~K}$ ), while probe pulses were 55 -fs pulses at $4.51 \mathrm{eV}$. $\rho$ was typically $3 \times 10^{17} \mathrm{~cm}^{-3}$. Pump and probe pulses, with a preset time delay $\Delta t$, were aligned coaxially and focused on the sample surfaces at $45^{\circ}$ to normal. For photoemission spectroscopy, images of the photoelectrons were recorded, using a hemispherical electron analyzer, as functions of energy and emission angle $\theta$ along the [001] crystallographic direction; surface normal emission was along [110]. The instrumental energy resolution was $50 \mathrm{meV}$, while the angle resolution was in the range of $\pm 1^{\circ}$.

The calculations were performed within density functional perturbation theory [11]. The band structure of GaAs was described within the local density approximation and pseudopotential method as in Refs. [9] and [10]. The topology of 


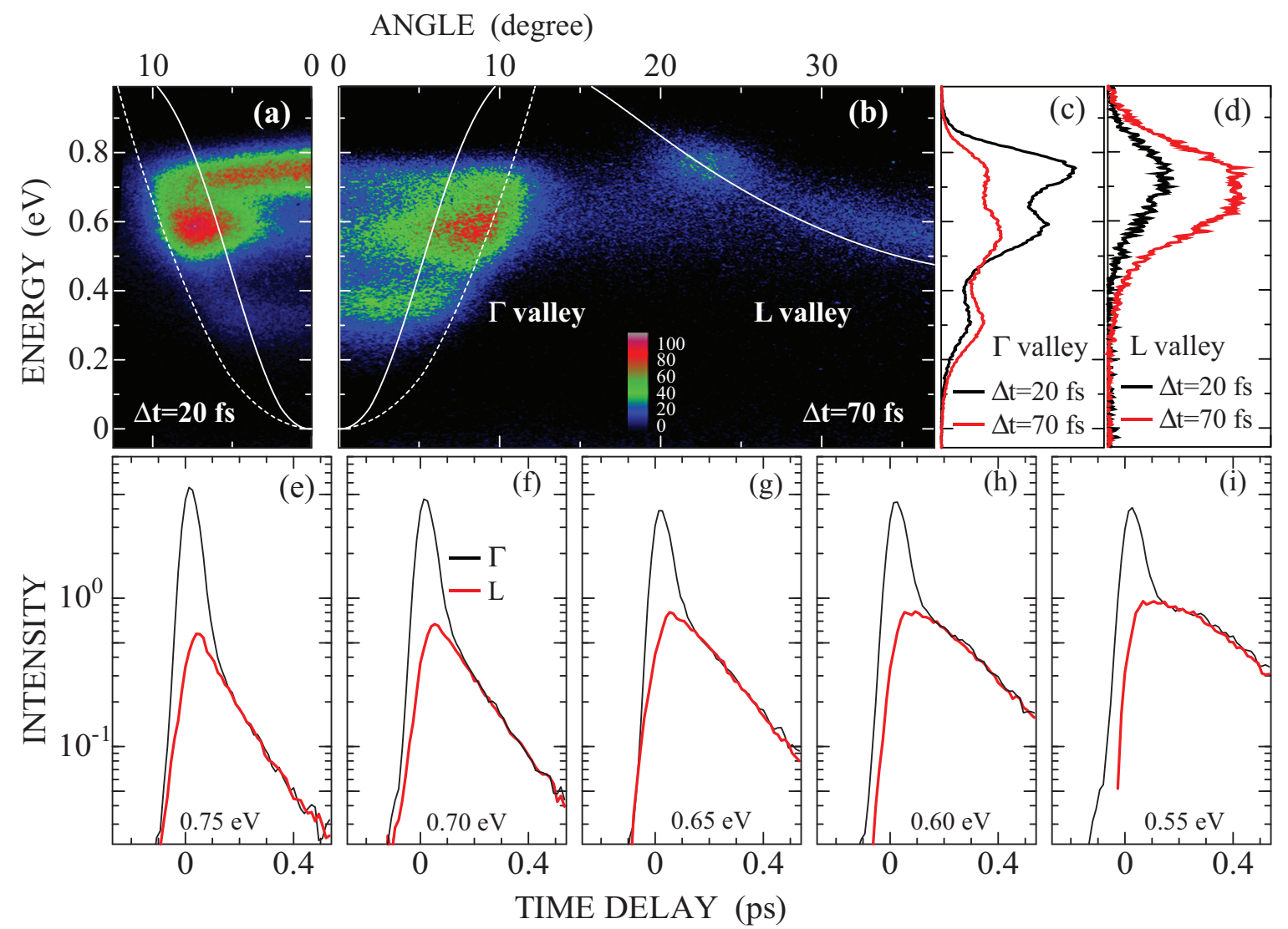

FIG. 1. (a) Photoemission image of nascent hot-electron distribution in the CB mapped in energy and momentum spaces 20 fs after excitation by $s$-polarized 2.30-eV light pulses. (b) Image of the hot-electron distribution $70 \mathrm{fs}$ after excitation in energy and momentum spaces including part of the $L$ valley $\left(\theta>13^{\circ}\right.$ ). In (a) and (b), the color scale indicates the photoemission intensity. Photoelectron energies are referenced to the conduction-band minimum, and solid and dashed curves show the band dispersion along $\Gamma-L$ and $\Gamma-X$ directions, plotted as a function of $\theta$, based on the present band-structure calculation. (c) Angle-integrated, energy-resolved, hot-electron distributions in the $\Gamma$ valley 20 and $70 \mathrm{fs}$ after excitation. (d) Angle-integrated, energy-resolved, hot-electron distributions in the $L$ valley 20 and $70 \mathrm{fs}$ after excitation. Even at $20 \mathrm{fs}$, the population of the $L$ valley is not negligible because of finite pulse widths and ultrafast scattering rates. (e)-(i) Energy-resolved dynamics of the populations in the $\Gamma$ (black) and $\mathrm{L}$ (red) valleys at excess energies ranging from 0.75 to $0.55 \mathrm{eV}$, respectively. For the sake of clarity, the $L$-valley intensities are multiplied by constants to have the same levels of intensities as those in the $\Gamma$ valley.

the conduction band is quantitatively well reproduced with the lattice parameter $a=10.49$ a.u. (see the Supplemental Material [12]). The $e$-ph matrix elements were first calculated on a $6 \times 6 \times 6$ q-point grid in the Brillouin zone, and then Fourier-interpolated on denser $48 \times 48 \times 48$ and $96 \times 96 \times 96 \mathbf{q -}$ point grids randomly shifted with respect to the zone center, using a recently developed interpolation method applicable to polar semiconductors $[10,18]$.

Figure 1(a) displays the distribution of hot electrons injected into the $\mathrm{CB}$ by $s$-polarized laser pulses with pump-photon energy $h v_{\text {pump }}=2.30 \mathrm{eV}$ at $\Delta t=20 \mathrm{fs}$. Energetically, three peaks are resolved, which correspond to optical transitions from the heavy-hole, light-hole, and split-off valence bands [6]. The directly captured distributions are highly localized in the momentum space. At $\Delta t=70 \mathrm{fs}$, the packets in the $\Gamma$ valley are partly dissipated, while a substantial amount of electrons has been transferred to the $L$ valley $\left(\theta>13^{\circ}\right)$. In Figs. 1(e)-1(i), temporal changes in the energy-resolved populations are reported both for the $\Gamma$ and $L$ valleys, for $\varepsilon$ ranging from 0.75 to $0.55 \mathrm{eV}$. The decay of the population in the $\Gamma$ valley is bimodal; an initial ultrafast decay with a time constant $\tau_{m}$ of the order of $10 \mathrm{fs}$ is followed by a slowly decaying component with a time constant of a few hundreds of fs, the magnitude of which depends on $\varepsilon$. The $\tau_{m}$ is identical to the growth time of the population in the $L$ valley, characterizing the delocalization in momentum space of the nascent distribution in the $\Gamma$ valley. Importantly, the slowly decaying component at a given $\varepsilon$ in the $\Gamma$ valley shows exactly the same decay characteristics as that in the $L$ valley for nonthermal and relaxing hot electrons within a wide range of $\varepsilon$.

For nonequilibrated hot electrons at each valley, phonon modes responsible for the intravalley relaxation, as well as the density of final states available inside each valley, are significantly different [19]; the intravalley relaxation times in the different valleys cannot be identical for a wide energy range. Therefore, the results in Fig. 1 show that the populations at $\Gamma$ and $L$ valleys are quasiequilibrated in the momentum space to give a common energy relaxation time $\tau_{E}$ at any valleys (see the Supplemental Material [12]). A concept similar to the momentum quasiequilibration among hot electrons at different valleys has been proposed by Stanton and Bailey 


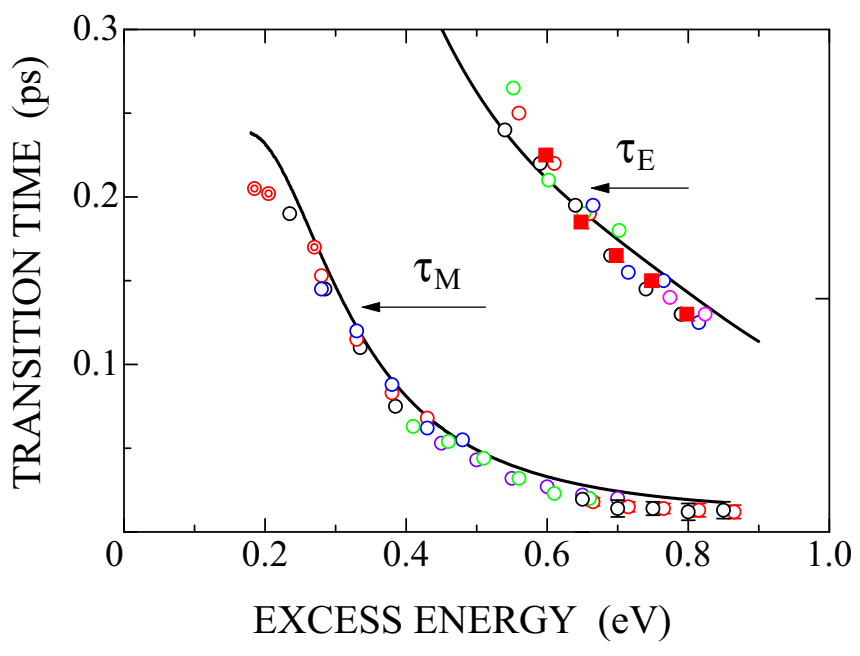

FIG. 2. Experimental and theoretical results of the momentum relaxation time $\tau_{m}$ and the energy relaxation time $\tau_{E}$ as a function of $\varepsilon$ at $293 \mathrm{~K}$. Different open symbols correspond to results obtained by the analysis of experimental data under different $h v_{\text {pump }}$ values. Solid red squares for $\tau_{E}$ show the results obtained from population changes in the $L$ valley shown in Figs. 1(e)-1(i). Solid curves show the theoretical results for $\tau_{m}$ and $\tau_{E}$ (see text).

in their analysis of intervalley scattering rates using simplified rate-equation models [20]. Our momentum-resolved spectroscopy has proven unambiguously that the momentum quasiequilibration is established within $150 \mathrm{fs}$ of excitation for hot electrons with $\varepsilon$ larger than $0.5 \mathrm{eV}$. Thus, the decay of the packet formed in the $\Gamma$ valley in GaAs leads to the formation of a hot-electron ensemble (HEE), which is quasiequilibrated in the momentum space (but not in energy space), via ultrafast momentum scattering.

We determined the magnitude of $\tau_{m}$ from temporal changes in the $\Gamma$-valley populations at a given $\varepsilon$ (with width of $\pm 25 \mathrm{meV}$ ), using the optical Bloch equation (OBE) [21-25], with population decay time $T_{1}\left(T_{1}=\tau_{m}\right)$ [24] and dephasing time $T_{2}$ which is given as $1 / T_{2}=1\left(2 T_{1}\right)+I / T_{2}^{*} ; T_{2}^{*}$ is a pure dephasing time (see the Supplemental Material of Ref. [6]). $\tau_{E}$ was determined by semilogarithmic plots as in Fig. 1 . The values of $\tau_{m}$ and $\tau_{E}$ at $293 \mathrm{~K}$ are displayed as a function of $\varepsilon$ in Fig. 2. Both become shorter with increasing $\varepsilon$ above the $L$-valley minimum. In particular, at $\varepsilon>0.4 \mathrm{eV}, \tau_{m}$ becomes as short as a few tens of fs.

In order to reveal the interaction responsible for the ultrafast momentum scattering [26], we studied the effects of $\rho$ and of temperature at several $h v_{\text {pump }}$ values. Under a $2.07-\mathrm{eV}$ excitation, for example, temporal changes in high-energy populations with $\varepsilon>0.5 \mathrm{eV}$ in the $\Gamma$ valley were examined at $\rho$ from $2.7 \times 10^{17}$ to $4.0 \times 10^{18} \mathrm{~cm}^{-3}$ at $293 \mathrm{~K}$ (see the Supplemental Material [12]). The magnitude of $\tau_{m}(=19 \pm 4 \mathrm{fs})$ is almost identical at any $\rho$. At variance, $T_{2}^{*}$ increases with increasing $\rho$, but it is much longer than $T_{2}$ in the present density regime, typically $\rho=3 \times 10^{17} \mathrm{~cm}^{-3}$ [27].

In contrast, the magnitude of $\tau_{m}$ changes drastically with changing temperature. Figure 3(a) displays the photoemission spectra, measured at time delays when the highest-energy peaks from the heavy-hole band show the maximum intensities at $e \simeq 0.5 \mathrm{eV}$ at 90 and $293 \mathrm{~K}$. Figure 3(b) shows temporal

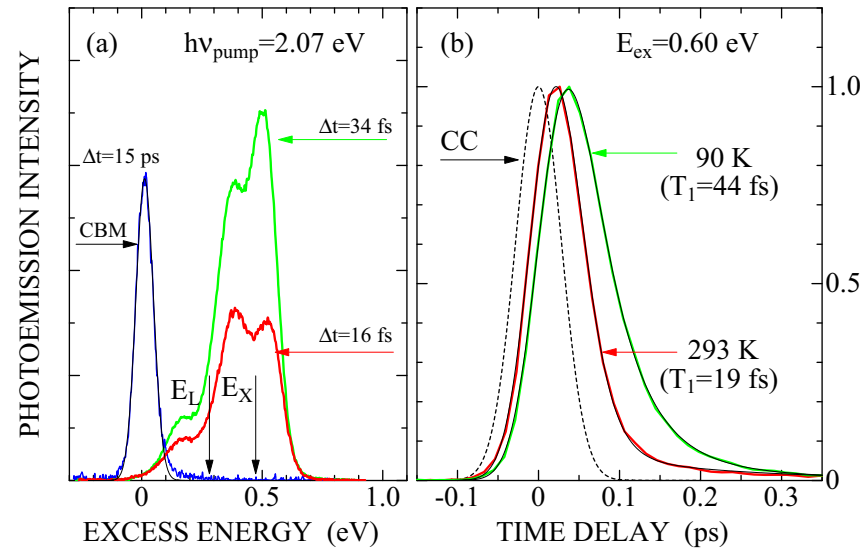

FIG. 3. (a) Photoemission spectrum measured at $90 \mathrm{~K}(\Delta t=$ $\left.34 \mathrm{fs}, h v_{\text {pump }}=2.14 \mathrm{eV}\right)$ [green (gray)], and that at $293 \mathrm{~K}(\Delta t=$ $16 \mathrm{fs}, h v_{\text {pump }}=2.07 \mathrm{eV}$ ) [red (dark gray)]. The blue (gray) spectrum labeled CBM shows the photoemission peak 15 ps after the excitation at $90 \mathrm{~K}$, from which the energy of the CBM is calibrated precisely by a convolved Boltzmann distribution at the CBM (thin solid curve). The temperature-dependent shift of the band-gap energy has been corrected to give the excess energy of hot electrons at both temperatures. (b) Temporal changes in photoemission intensities at the higher-energy side of the highest-energy peak in (a).

changes of the intensities at $\varepsilon=0.60 \mathrm{eV}$, the high-energy side of the highest-energy peaks. Analysis using OBE shows that the population decay time is $44 \pm 3 \mathrm{fs}$ at $90 \mathrm{~K}$, while it is $19 \pm 4 \mathrm{fs}$ at $293 \mathrm{~K}$. Therefore, the magnitude of $\tau_{m}$ is strongly temperature dependent, revealing the crucial role of the $e$-ph interaction in the ultrafast momentum delocalization at $\rho<5 \times 10^{17} \mathrm{~cm}^{-3}$.

We calculate $e$-ph scattering rates from first principles using the recently developed method which takes into account the polar optical phonon (POP) interaction appropriately in polar materials [10]. For an electronic state $|n, \mathbf{k}\rangle$ with eigenenergy $\varepsilon_{n, \mathbf{k}}$ in the band $n$ with the wave vector $\mathbf{k}$ in the $\Gamma$ valley, the total probability $\Gamma_{n, \mathbf{k}}$ of $e$-ph scattering is defined as a sum of absorption and emission terms [28,29]:

$$
\begin{aligned}
\Gamma_{n, \mathbf{k}}^{\mathrm{abs} / \mathrm{em}}= & \int_{\mathrm{BZ}} d^{3} \mathbf{q} \sum_{n^{\prime}} \sum_{\lambda} \frac{2 \pi}{\hbar}\left|\left\langle n^{\prime}, \mathbf{k} \pm \mathbf{q}\left|\Delta W_{\mathbf{q}}^{\lambda}\right| n, \mathbf{k}\right\rangle\right|^{2} \\
& \times \mathcal{L}\left(\varepsilon_{n, \mathbf{k}}-\varepsilon_{n^{\prime}, \mathbf{k} \pm \mathbf{q}} \pm \hbar \omega_{\mathbf{q}}^{\lambda} ; 2 \gamma\right)\left\{\begin{array}{c}
N_{\mathbf{q}}^{\lambda}(T) \\
N_{\mathbf{q}}^{\lambda}(T)+1
\end{array}\right\},
\end{aligned}
$$

where $\Delta W_{\mathbf{q}}^{\lambda}$ is the crystal-potential variation induced by the unit amplitude of vibration of a phonon mode $\lambda$ and a wave vector $\mathbf{q}$ ( $\hbar \omega_{\mathbf{q}}^{\lambda}$ being the phonon energy smaller than $36 \mathrm{meV})$, and $N_{\mathbf{q}}^{\lambda}(T)$ is the phonon occupation number. The intrinsic broadening $\gamma$ of the initial and final electronic states is described by a Lorentzian function $\mathcal{L}[19,29]$.

As both $\Gamma_{n, \mathbf{k}}^{\mathrm{abs}}$ and $\Gamma_{n, \mathbf{k}}^{\mathrm{em}}$ contain momentum scatterings of a state $|n, \mathbf{k}\rangle$ by finite-wave-vector phonons, we take their sum $\Gamma_{n, \mathbf{k}}$ as a measure of the momentum scattering rate. We calculated Eq. (1) self-consistently to have $\gamma=\Gamma_{n, \mathbf{k}}[29,30]$. Computed $\Gamma_{n, \mathbf{k}}$ are plotted in Fig. 4(a), together with the scattering rates into $L, X$, and $\Gamma$ valleys [31-33]. The rates are enhanced drastically in the high- $\varepsilon$ regions, which comes from an increase of the density of final states at the $L$ and 


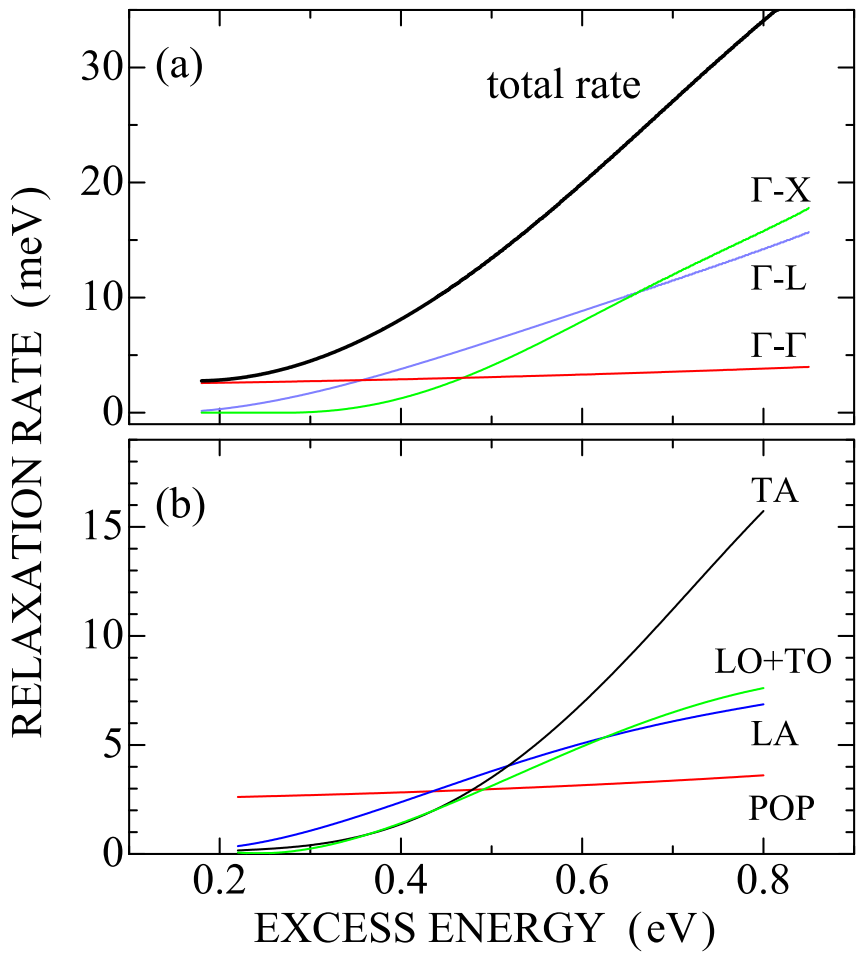

FIG. 4. (a) Theoretical results of momentum relaxation rate with contributions of different scattering channels to the total rate. The total rate is shown in black. Blue (gray) line: $\Gamma-L$ intervalley scattering. Green (light gray) line: $\Gamma-X$ intervalley scattering. Red (dark gray) line: intravalley scattering within the $\Gamma$ valley. (b) The contributions of different phonon modes to the momentum relaxation rate for an initial electronic state in the $\Gamma$ valley. Black line: contribution of transverse-acoustic (TA) modes. Blue (gray) line: contribution of the longitudinal-acoustic (LA) mode. Green (light gray) line: contribution due to optical modes [excluding the interaction with polar optical phonons (POPs)]. Red (dark gray) line: contribution due to the POP interaction calculated solely with the method presented in Ref. [10].

$X$ valleys. Figure 4(b) shows relative contributions by respective phonon modes to $\Gamma_{n, \mathbf{k}}$. Longitudinal-acoustic- (LA-) and transverse-acoustic- (TA-) phonon modes induce intervalley scattering predominantly, and TA modes contribute to enhance the $\Gamma-X$ scattering $[9,34]$.

The theoretical results for $\tau_{m}$, defined as $\tau_{m}\left(\varepsilon_{n, \mathbf{k}}\right)=\hbar / \Gamma_{n, \mathbf{k}}$, are compared with experimental ones in Fig. 2. Not only does the dependence on $\varepsilon$ agree very well with the experimental results, but so does the absolute magnitude of $\tau_{m}$, asserting that the ultrafast momentum scattering of the hot-electron packets is due to the $e$-ph interaction. Importantly, the experimentally found temperature dependence of $\tau_{m}$ in Fig. 3 is also fully explained by the $a b$ initio calculation: The theoretical $\tau_{m}$ of $19.6 \mathrm{fs}$ at $300 \mathrm{~K}$ becomes $44.9 \mathrm{fs}$ at $90 \mathrm{~K}$ for $\varepsilon=0.79 \mathrm{eV}$, substantiating the theoretical method used.

Calculating $\tau_{E}$ of HEEs spread over a whole BZ is challenging, as all possible $e$-ph scattering processes can, in principle, contribute to the energy loss at a given $\varepsilon$. We considered the difference $\Gamma_{n, \mathbf{k}}^{\mathrm{em}}-\Gamma_{n, \mathbf{k}}^{\mathrm{abs}}$ (see Supplemental Material [12]), together with the effective frequencies of emitted (absorbed) phonons for all possible processes [35]. The solid line in Fig. 2 is the theoretical result, showing excellent agreement with the experimental data. We emphasize that the magnitude and dependence on $\varepsilon$ of $\tau_{E}$ can be captured only when energyloss processes by finite-wave-vector phonons are correctly accounted for; the POP interaction in the $\Gamma$ and $L$ valleys plays a minor role [12]. Thus, our results demonstrate a significant difference in the energy-relaxation mechanism for highly excited hot electrons with respect to hot electrons near the $\mathrm{CB}$ minimum $(\mathrm{CBM})$ in the $\Gamma$ valley.

In conclusion, we have unveiled the unique characteristics of two-step dynamics in the ultrafast relaxation of highly excited hot electrons photoinjected into the $\Gamma$ valley in GaAs. The mechanism is applicable to other crystalline solids with multivalley band structures.

This work was supported by the Japan Society for the Promotion of Science (JSPS) KAKENHI Grant No. 24000006. Calculations were performed with the QUANTUM ESPRESSO [11,36] and WANNIER 90 packages. We acknowledge support from the DGA. Computer time has been granted by GENCI (Project No. 2210) and by Ecole Polytechnique through the LLR-LSI project.
[1] J. Shah, Ultrafast Spectroscopy of Semiconductors and Semiconductor Nanostructures, 2nd ed. (Springer, Berlin, 1999).

[2] F. Rossi and T. Kuhn, Theory of ultrafast phenomena in photoexcited semiconductors, Rev. Mod. Phys. 74, 895 (2002).

[3] V. M. Axt and T. Kuhn, Femtosecond spectroscopy in semiconductors: A key to coherences, correlations and quantum kinetics, Rep. Prog. Phys. 67, 433 (2004), and references therein.

[4] M. Joschko, M. Woerner, T. Elsaesser, E. Binder, T. Kuhn, R. Hey, H. Kostial, and K. Ploog, Heavy-Light Hole Quantum Beats in the Band-to-Band Continuum of GaAs Observed in 20 Femtosecond Pump-Probe Experiments, Phys. Rev. Lett. 78, 737 (1997).

[5] O. Schubert, M. Hohenleutner, F. Langer, B. Urbanek, C. Lange, U. Huttner, D. Golde, T. Meier, M. Kira, S. W. Koch, and R. Huber, Sub-cycle control of terahertz high-harmonic generation by dynamical Bloch oscillations, Nat. Photon. 8, 119 (2014).
[6] J. Kanasaki, H. Tanimura, and K. Tanimura, Imaging Energy-, Momentum-, and Time-resolved Distributions of Photoinjected Hot Electrons in GaAs, Phys. Rev. Lett. 113, 237401 (2014).

[7] H. Tanimura, J. Kanasaki, and K. Tanimura, Ultrafast scattering processes of hot electrons in InSb studied by time- and angleresolved photoemission spectroscopy, Phys. Rev. B 91, 045201 (2015).

[8] J. Sjakste, I. Timrov, P. Gava, N. Mingo, and N. Vast, in Annual Review of Heat Transfer (Begell House, Danbury, CT, 2014), Vol. 17, p. 333, and references therein.

[9] J. Sjakste, N. Vast, and V. Tyuterev, Ab initio Method for Calculating Electron-Phonon Scattering Times in Semiconductors: Application to GaAs and GaP, Phys. Rev. Lett. 99, 236405 (2007).

[10] J. Sjakste, N. Vast, M. Calandra, and F. Mauri, Wannier interpolation of the electron-phonon matrix elements in polar 
semiconductors: Polar-optical coupling in GaAs, Phys. Rev. B 92, 054307 (2015).

[11] S. Baroni, S. de Gironcoli, A. D. Corso, and P. Giannozzi, Phonons and related crystal properties from density-functional perturbation theory, Rev. Mod. Phys. 73, 515 (2001).

[12] See Supplemental Material at http://link.aps.org/supplemental/ 10.1103/PhysRevB.93.161203 for Conduction-band structure within the density functional theory, Energy-relaxation time depending only on the excess energy, Results of excitationdensity dependent changes in ultrafast population loss, and details of calculation of the energy relaxation rate, which includes Refs. [13-17].

[13] I. Saïdi, S. Ben Radhia, and K. Boujdaria, Band parameters of GaAs, InAs, InP, and InSb in the 40-band $\mathbf{k} \cdot \mathbf{p}$ model, J. Appl. Phys. 107, 043701 (2010).

[14] S. Richard, F. Aniel, and G. Fishman, Energy-band structure of $\mathrm{Ge}, \mathrm{Si}$, and GaAs: A thirty-band $\mathbf{k} \cdot \mathbf{p}$ method, Phys. Rev. B 70, 235204 (2004); 71, 169901(E) (2005).

[15] C. J. Stanton and D. W. Bailey, in Evaluating Photoexcitation Experiments Using Monte Carlo Simulations in Monte Carlo Device Simulation: Full Band and Beyond, edited by K. Hess (Kluwer Academic, Boston, 1991).

[16] P. C. Becker, H. L. Fragnito, C. H. Brito Cruz, R. L. Fork, J. E. Cunningham, J. E. Henry, and C. V. Shank, Femtosecond Photon Echoes from Band-to-Band Transitions in GaAs, Phys. Rev. Lett. 61, 1647 (1988).

[17] P. Vogl, Microscopic theory of electron-phonon interaction in insulators or semiconductors, Phys. Rev. B 13, 694 (1976).

[18] A development similar to Ref. [10] was described in C. Verdi and F. Giustino, Fröhlich Electron-Phonon Vertex from First Principles, Phys. Rev. Lett. 115, 176401 (2015).

[19] B. K. Ridley, Quantum Processes in Semiconductors (Oxford University Press, Oxford, UK, 1999).

[20] C. J. Stanton and D. W. Bailey, Rate equations for the study of femtosecond intervalley scattering in compound semiconductors, Phys. Rev. B 45, 8369 (1992).

[21] N. Bloembergen, Nonlinear Optics (Benjamin, New York, 1965).

[22] M. Schubert and B. Wilhelmi, Nonlinear Optics and Quantum Electronics (John Wiley \& Sons., New York, 1986).

[23] T. Hertel, E. Knoesel, M. Wolf, and G. Ertl, Ultrafast Electron Dynamics at $\mathrm{Cu}(111)$ : Response of an Electron Gas to Optical Excitation, Phys. Rev. Lett. 76, 535 (1996).

[24] M. Bauer, S. Pawlik, and M. Aeschlimann, Decay dynamics of photoexcited alkali chemisorbates: Real-time investigations in the femtosecond regime, Phys. Rev. B 60, 5016 (1999).

[25] M. J. Weida, S. Ogawa, H. Nagano, and H. Petek, Ultrafast interferometric pumpprobe correlation measurements in systems with broadened bands or continua, J. Opt. Soc. Am. B 17, 1443 (2000).
[26] M. J. Kann, A. M. Kriman, and D. K. Ferry, Effect of electronelectron scattering on intervalley transition rates of photoexcited carriers in GaAs, Phys. Rev. B 41, 12659 (1990).

[27] The dephasing time $T_{2}$ under 2-eV light excitation has been determined by photon-echo experiments; it is 31.2 fs at $\rho=$ $3.5 \times 10^{17} \mathrm{~cm}^{-3}$ in Ref. [16]. Based on the result that the magnitude of $T_{2}$ decreases with increasing $\rho, T_{2}$ has been ascribed entirely to the $e$-e interaction. However, $T_{1}$ at $h v_{\text {pump }}$ is as short as $19 \mathrm{fs}$, giving the predominant contribution to the dephasing rate of $1 / T_{2}$ in the low- $\rho$ regime. The possible contribution of the $e$-ph interaction to the polarization dephasing has been pointed out in Ref. [26].

[28] C. Jacoboni and L. Reggiani, The Monte Carlo method for the solution of charge transport in semiconductors with applications to covalent materials, Rev. Mod. Phys. 55, 645 (1983).

[29] S. Piscanec, M. Lazzeri, J. Robertson, A. C. Ferrari, and F. Mauri, Optical phonons in carbon nanotubes: Kohn anomalies, Peierls distortions, and dynamic effects, Phys. Rev. B 75, 035427 (2007).

[30] Our calculations were carefully converged with respect to the q-point grid and broadening $\gamma$. For $\varepsilon_{n, \mathbf{k}}>0.38 \mathrm{eV}$ with respect to the CBM, the calculated electron-phonon widths $\Gamma_{n, \mathbf{k}}$ were found inside the convergence region for $10 \mathrm{meV}<2 \gamma<$ $60 \mathrm{meV}$, therefore the calculations can be regarded as selfconsistent, $\gamma=\Gamma_{n, \mathbf{k}}$. For smaller excess energies we used the smallest broadening corresponding to numerical convergence of our integrals: $2 \gamma=10 \mathrm{meV}$.

[31] It must be noted that due to the relatively weak contribution of POP to $\Gamma_{n, \mathbf{k}}$ (Fig. 4), our theoretical results are similar to the recent calculations of Ref. [32], notwithstanding the neglect of POP coupling in the latter work (see Ref. [10]). A calculation similar to Ref. [32] has been performed for silicon in Ref. [33].

[32] M. Bernardi, D. Vigil-Fowler, C. S. Ong, J. B. Neaton, and S. G. Louie, Ab initio study of hot electrons in GaAs, Proc. Natl. Acad. Sci. USA 112, 5291 (2015).

[33] M. Bernardi, D. Vigil-Fowler, J. Lischner, J. B. Neaton, and S. G. Louie, $A b$ initio Study of Hot Carriers in the First Picosecond after Sunlight Absorption in Silicon, Phys. Rev. Lett. 112, 257402 (2014).

[34] S. Zollner, J. Kircher, M. Cardona, and S. Gopalan, Are transverse phonons important for $\Gamma$-X-intervalley scattering? Solid State Electron. 32, 1585 (1989).

[35] V. P. Zhukov, P. M. Echenique, and E. V. Chulkov, Two types of excited electron dynamics in zinc oxide, Phys. Rev. B 82, 094302 (2010).

[36] P. Giannozzi, S. Baroni, N. Bonini, M. Calandra, R. Car, C. Cavazzoni, D. Ceresoli, G. Chiarotti, M. Cococcioni, I. Dabo, A. Dal Corso, S. de Gironcoli, S. Fabris, G. Fratesi, R. Gebauer, U. Gerstmann, C. Gougoussis, A. Kokalj, M. Lazzeri, L. MartinSamos et al., QUANTUM ESPRESSO: A modular and opensource software project for quantum simulations of materials, J. Phys.: Condens. Matter 21, 395502 (2009). 